\title{
VI. An electromagnetic theory of radiation
}

\section{H. Bateman}

To cite this article: $\mathrm{H}$. Bateman (1921) Vl. An electromagnetic theory of radiation, Philosophical Magazine Series 6, 41:241, 107-113, DOI: 10.1080/14786442108636200

To link to this article: http://dx.doi.org/10.1080/14786442108636200

曲 Published online: 08 Apr 2009.

6 Submit your article to this journal $\pi$

Џll Article views: 5

Q View related articles $₫$ 


\section{$\left[\begin{array}{ll}107 & ]\end{array}\right.$ \\ VI. An Electromagnetic Theory of Rudiution. By H. Bateman *.}

1. COME interesting suggestions with regard to the nature $D$ of radiation have been made recently by Sir Joseph Thomson $\dagger$ and Leigh Page $\ddagger$. According to the first writer, radiation of energy seems to be generally associated with the formation of closed lines of electric force which travel awar from a source with the velocity of light. In Page's work radiation of energy is supposed to be caused by a rotation of the field of an electron, and it follows from his expressions for the field vectors in a rotating field that this field may be obtained by superposing on the non-rotating field of an electron a radiant field in which circular lines of electric force travel outwards with the velocity of light.

Page's work is particularly interesting because the amount of energy radiated in one complete revolution of the field is almost exactly $\frac{1}{3-2} h \nu$, where $v$ is the frequency of the rotation and $h$ is Planck's constant. In order that $\nu$ may be identified with the frequency of the emitted light, it seems necessary either to assume the existence of discrete tubes of force or to extend Page's analysis to the case in which an electron revolves or oscillates with its frequency of rotation.

A peculiar feature of the radiant field is that there is a radiation of magnetic charges which travel along straight lines with the velocity of light $\S$. There is, however, no magnetic charge associated with the electron, and so it seems likely that the magnetic charges arise from a distribution of magnetic doublets which in turn arise from moving electric doublets. Whether this is the case or not, the mathematical analysis suitable for the formal development of this theory of radiation is akin to that which has been given in the present author's speculations regarding the electrical nature

* Communicated by the Author.

+ 'Engineering, vol. 101. p. 381 (1916); Phil. Mag. June 1920, p. 679.

† Proc. Nat. Acad. Sci. March 1920, p. 115.

$\$ A$ class of radiant fields in $w$ hich there is a radidtion of magnetic particles in particular directions was found by the present anthor, Proc. Nat. Acad. Sci. March 1918. It is probable that Page's radiant field can be built up by superposing a number of fields of this older type. It may be remarked, too, that circular lines of electric force can be obtained in fields of the older type, and in this case it is possible for marnetic charges which are equal but of opposite sign to be emitted in consecutive directions. Circular lines of electric force of opposite senses are then generated in approximately the same regiou like vortices having different senses of rotation. 
of the xther, and so it may be worth while to present the analysis in a compact form.

2. In a former paper ${ }^{*}$ an attempt was made to describe the motion of the lines of force of an electric pole by means of a succession of infinitesimal transformations which transform light-particles into light-particles. If for simplicity we consider only transformations which correspond to rigid body-displacements in Minkowski's four-dimensional space, the path curves or trajectories of the transformations may be written in the form

$$
\left.\begin{array}{rl}
d x & =d \tau\left[\xi^{\prime}(\tau)+c(t-\tau) p-(y-\eta) h+(x-\zeta) g\right], \\
d y & =d \tau\left[\eta^{\prime}(\tau)+c(t-\tau) q-(x-\zeta) f^{\prime}+(x-\xi) h\right], \\
d z & =d \tau\left[\zeta^{\prime}(\tau)+c(t-\tau) r-(x-\xi) g+(y-\eta) f^{\prime}\right], \\
c d t & =d \tau[c+(x-\xi) p+(y-\eta) q+(x-\zeta) r],
\end{array}\right\}
$$

where $\xi, \eta, \zeta, f, g, h, p, q, r$ are functions of $\tau$. The infinitesimal transformation associated with a particular value of $\boldsymbol{\tau}$ is supposed to be applied to space-time points $(x, y, z, t)$ which satisfy the relation

$$
[x-\xi(\tau)]^{2}+[y-\eta(\tau)]^{2}+[z-\zeta(\tau)]^{2}=c^{2}(t-\tau)^{2},
$$

and also to certain space-time points specified by equations of type

$$
\left.\begin{array}{l}
x=\mathrm{F}_{1}(\mathrm{X}, \mathrm{Y}, \mathrm{Z}, \tau), \quad z=\mathrm{F}_{3}(\mathrm{X}, \mathrm{Y}, \mathrm{Z}, \tau), \\
y=\mathrm{F}_{2}(\mathrm{X}, \mathrm{Y}, \mathrm{Z}, \tau), \quad t=\mathrm{F}_{0}(\mathrm{X}, \mathrm{Y}, \mathrm{Z}, \tau),
\end{array}\right\} .
$$

which may be regarded as solutions of the differential equations (1), X, Y, and Z being constants of integration or invariants for the sequence of transformations.

The equations (3) may be supposed to give the co-ordinated motions of the different points of an electron of which $(\xi, \eta, \zeta, \pi)$ is a particular point wrich we shall call the focus. The lines of force of the electron are supposed to be generated by light-particles fired out from the different positions of this focus $S$, the direction of projection of the light-particles associated with one line of force varying in a manner indicated by the succession of infinitesimal transformations. In fact, if $(l, m, n)$ are the direction cosines of the line of projection of the light-particles emitted at time $\tau$ we have

$$
\frac{d l}{d \tau}=p-m h+n g-l(l p+m q+n r) . . . .
$$

* Proc. London Math. Soc. (2) vol. xүiii. p. 95 (1919). 
3. Let us now consider the electromagnetic field specified by the vectors $\mathbf{E}$ and $\mathbf{H}$ whose components are given by equations of type

$$
\begin{aligned}
& \mathrm{E}_{x}=\frac{1}{c} \frac{\partial(\sigma, \tau)}{\partial(x, t)}+\frac{\partial(\theta, \tau)}{\partial(y, z)}, \quad \mathrm{H}_{x}=\frac{\partial(\sigma, \tau)}{\partial(y, z)}-\frac{1}{c} \frac{\partial(\theta, \tau)}{\partial(x, t)}, \\
& \left.\begin{array}{l}
\mathrm{M} \sigma=(x-\xi) \alpha+(y-\eta) \beta+(z-\zeta) \gamma+\delta, \\
\mathrm{M} \theta=(x-\xi) \lambda+(y-\eta) \mu+(z-\zeta) \nu, \\
\mathrm{M}=(x-\xi) \xi^{\prime}+(y-\eta) \eta^{\prime}+(z-\zeta) \zeta^{\prime}-c^{2}(t-\tau)
\end{array}\right\}
\end{aligned}
$$

where $\alpha, \beta, \gamma, \delta, \lambda, \mu, \nu$ are functions of $\tau$, and $c$ is the velocity of light. Writing

$$
\frac{x-\xi}{l}=\frac{y-\eta}{m}=\frac{z-\zeta}{n}=\frac{c(t-\tau)}{1},
$$

we see that if

$$
\begin{aligned}
& \delta_{d \tau}^{d l}=c \alpha+\mu \zeta^{\prime}-\nu \eta^{\prime}+m\left(c \nu-\alpha \eta^{\prime}+\beta \xi^{\prime}\right)-n\left(c \mu-\gamma \xi^{\prime}+\alpha \zeta^{\prime}\right) \\
& \quad-l\left[l\left(c \alpha+\mu \zeta^{\prime}-\nu \eta^{\prime}\right)+m\left(c \beta+\nu \xi^{\prime}-\lambda \xi^{\prime}\right)+n\left(c \gamma+\lambda \eta^{\prime}-\mu \xi^{\prime}\right)\right] .
\end{aligned}
$$

we may write

$$
\mathbf{E}=\frac{1}{c} \frac{\partial \tau}{\partial t} \frac{\delta}{\mathbf{M}^{2}}\left[c \mathbf{s}-\mathbf{\nabla}-c(t-\tau) \mathbf{s}^{\prime}\right], \quad \mathbf{H}=\mathbf{s} \times \mathbf{E}, \quad .
$$

where a, w, v, and s denote the vectors with components. $(\alpha, \beta, \gamma),(\lambda, \mu, \nu),\left(\xi^{\prime}, \eta^{\prime}, \zeta^{\prime}\right),(l, m, n)$ respectively.

The rate of radiation of energy in the direction $s$ at a very great distance from $\mathrm{S}$ is approximately

$$
\frac{c}{4 \pi} \delta_{\overline{\mathbf{M}^{4}}}\left(\frac{\partial \tau}{\partial t}\right)^{2}(t-\tau)^{2}\left(l^{\prime 2}+m^{2}+n^{\prime 2}\right),
$$

and this is positive except when $\mathrm{s}$ does not vary with $\tau$. Both $a$ and $\mathbf{w}$ must be zero for there to be no radiation.

The equation (7) is identical with (4) if

$$
c \alpha+\mu \zeta^{\prime}-\nu \eta^{\prime}=\delta \dot{p}, \quad c \lambda-\beta \zeta^{\prime}+\gamma \eta^{\prime}=-\delta f .
$$

These equations generally determine the ratios of $\alpha, \beta, \gamma, \delta$, $\lambda, \mu, \nu$ uniquely in terms of $f, g, h, p, q, r$. To make the electric charge associated with $\mathrm{S}$ a constant quantity, $e$, we write

$$
4 \pi \delta=e\left(c^{2}-\xi^{\prime 2}-\eta^{\prime 2}-\zeta^{\prime 2}\right),
$$


and the field is then completely determined when the motion of the electron is known.

4. The part of the field depending on the function $\theta$ represents the radiant field added by Page to allow for the effect of rotation. A moving line of electric force in this field is given by the equations $\theta=$ constant, $\tau=$ constant. When $t$ is constant the lines of electric force associated with a given value of $\tau$ are cut out on the sphere (2) by the planes through the polar line of a line which meets the sphere in two real points $A$ and $B$. The directions $S A$ and $S B$ are "associated" directions of projection, the points $A$ and $B$ being collinear with the point 'I' which would be reached by $S$ at time $t$ if it continued to move with the velocity which it has at the instant of lime $\tau$. As $t$ and $\tau$ vary the points $A$ and $B$ trace out two associated lines of electric force. These may be regarded as examples of the guiding lines of force of which Sir Joseph Thomson speaks. The other lines of force circle round one of these lines.

The lines of magnetic force being orthogonal to the lines of electric force are circles through pairs of points such as $A$ and $B$. It should be noticed that the field vectors in the radiant field may also be expressed in the form

where

$$
\mathrm{H}_{x}=\psi \frac{\partial(\phi, \tau)}{\partial(y, z)}, \quad \mathrm{E}_{x}=\frac{1}{c} \psi \frac{\partial(\phi, \tau)}{\partial(x, t)},
$$

$$
\begin{gathered}
\phi=\log \frac{\mathrm{P}(x-\xi)+\mathrm{Q}(y-\eta)+\mathrm{R}(z-\zeta)-\mathrm{S}(t-\tau)}{\mathrm{U}(x-\xi)+\mathrm{V}(y-\eta)+\mathrm{W}(z-\zeta)} \equiv \log \frac{\mathrm{A}}{\mathrm{B}}, \\
\mathrm{SU}=\eta^{\prime} \nu-\mu \zeta^{\prime}, \quad \mathrm{SV}=\zeta^{\prime} \lambda-\xi^{\prime} v, \quad \mathrm{SW}=\xi^{\prime} \mu-\eta^{\prime} \lambda, \\
\lambda=\mathrm{VR}-\mathrm{WQ}, \quad \mu=\mathrm{WP}-\mathrm{UR}, \quad \nu=\mathrm{UQ}-\mathrm{VP}, \\
\psi=\frac{\mathrm{AB}}{\mathrm{M}^{2}} .
\end{gathered}
$$

The lines of magnetic force are given by $\phi=$ constant, $\tau=$ constant, and are traced out by particles which travel along the radii for which $\frac{A}{M}, \frac{B}{M}$, and $\tau$ are constant. There are two such radii for each set of values of $\phi, \psi$, and $\tau$. The line joining the positions at time $t$ of the magnetic particles which travel along these radii passes through a fixed point $\mathrm{Q}(t, \tau)$ given by the equations $\mathrm{A}=0, \mathrm{~B}=0, \mathrm{M}=0$. The polar plane of $Q$ with respect to the sphere (2) divides the positive magnetic charges on the sphere from the negative 
magnetic charges. The volume density of the magnetic charge may be written in the form

$$
\epsilon=\frac{\partial(\psi, \phi, \tau)}{\partial(x, y, z)}=-\frac{2(t-\tau)}{\mathbf{M}^{4}}\left[\mathbf{M}(\mathbf{v} \cdot \mathbf{w})+\left(c^{2}-v^{2}\right) \theta\right],
$$

and this is equivalent to the expression given by Page when we write

$$
\mathbf{w}=-\frac{e c}{4 \pi}\left(1-\frac{v^{2}}{e^{2}}\right) \omega
$$

where $\omega$ is a vector representing the angular velocity of the electron or its field in the case when $v=0$.

5 . The rate of radiation of energy in a field of type (5) is found to be

$$
\frac{32}{3} c \pi^{2}\left[\frac{a^{2}+w^{2}-\frac{1}{c}\{\mathbf{v} \cdot(\mathbf{a} \times \mathbf{w})\}}{\left(c^{2}-v^{2}\right)^{2}}+\frac{(\mathbf{v} \cdot \mathbf{a})^{2}+(\mathbf{v} \cdot \mathbf{w})^{2}}{\left(c^{2}-v^{2}\right)^{3}}\right] .
$$

The vectors $a$ and $\mathbf{w}$ are at present at our disposal. If we take $a=0$ and choose the above value of $w$, assuming also that $(\mathbf{v} . \mathbf{w})=0$, we obtain for the rate of radiation

$$
\frac{2 e^{2} \omega^{2}}{3 c}
$$

and the amount of energy radiated in an interval $\frac{2 \pi}{\omega}$ is * $\frac{4 \pi e^{2} \omega}{3 e}$, which is approximately $\frac{\omega}{b 4 \pi} 6 \cdot 4(10)^{-27}$, when $e$. is assumed to be $4.77 \times 10^{-10}$ E.S.U.

6. The possibility of radiation in quanta having been established the next step must be to justify our assumption that $a=0$. When the electron is stationary or moving aniformly in a straight line this assumption seems quite natural, but there must be some occasion when an electron moves in a curve, and so we must consider the possibility that a may be zero in a general type of motion.

In the case of a moving electric pole the expression for $\sigma$ is $\dagger$

$$
\sigma=\frac{\left.e \xi^{\prime \prime}(x-\xi)+\eta^{\prime \prime}(y-\eta)+\zeta^{\prime \prime} \cdot z-\zeta\right)+e^{2}-\xi^{\prime 2}-\eta^{\prime 2}-\zeta^{\prime 2}}{4 \pi \quad \xi^{\prime}(x-\xi)+\eta^{\prime}(y-\eta)+\zeta^{\prime}(z-\zeta)-c^{2}(t-\tau)}+f^{\prime}(\tau) .
$$

* A similar result may be obtained without the introduction of magnetic particles by putting $w=0$ and regarding $a$ as a multiple of an andular velocity about an axis perpendicular to $v$.

* Cf. R. Hargreaves, Proc. Camb. Phil. Soe, 1815. 
Now by making a suitable choice of $f(\tau)$ this may be written in the form

$$
\begin{aligned}
& +\left\{\frac{d}{d \tau}\left(\frac{\partial \mathrm{L}}{\partial \bar{\zeta}^{\prime}}\right)\right\}(z-\zeta)+\frac{d}{d \tau}\left(\frac{c^{2} \mathrm{~L}}{c^{2}-v^{2}}\right)(t-\tau)-\mathrm{L} \\
& \sigma=\frac{e}{\frac{\left\{\frac{d}{d \tau}\left(\frac{\partial L}{\partial \xi^{\prime}}\right)\right\}(x-\xi)+\left\{\frac{d}{d \tau}\left(\frac{\partial L_{i}}{\partial \eta^{\prime}}\right)\right\}(y-\eta)}{\partial L}(x-\xi)+\frac{\partial L}{\partial \eta^{\prime}}(y-\eta)+\frac{\partial L}{\partial \zeta^{\prime}}(z-\zeta)+\frac{c^{2} L}{c^{2}-\imath^{2}}(t-\tau)},
\end{aligned}
$$

where $\mathrm{L}=m\left(c^{2}-v^{2}\right)^{\frac{1}{2}}$ reminds us of a Hamiltonian function used in the theory of relativity ${ }^{*}, m$ being a constant of the nature of mass.

If $\mathbf{a}$ is zero in the case of an electron this may be in consequence of the equations of motion of the electron and it may be necessary to extend the expression for $\sigma$ by adding to $\frac{d}{d \tau}\left(\frac{\partial L}{\partial \xi^{\prime}}\right)$ a term representing the effeet of the external electric field, and possibly also a term $-\frac{\partial \mathrm{L}}{\partial \xi}$ to allow for a variation of $m$ with position. The complete coefficient of $x-\xi$ is then zero on account of the equations of motion, and the complete coefficient of $t-\tau$ should be zero on account of the energy equation.

This modification of $\sigma$ makes the introduction of a radiant field depending on $\theta$ seem fairly reasonable, because the coefficients in the numerator of $\theta$ may be regarded as components of angular momentum when the denominator has been suitably modified. It is possible, too, that there should also be terms to represent the effect of impulsive couples. which act only at isolated intervals of time. These impulsive couples may, perhaps, be produced by magnetic particles emitted from other sources.

7. It is, perhaps, a little hard to understand why the electron should turn completely round a finite number of times when emitting radiation. It may be that a particular line of force is naturally associated with some particle at a very great distance from $S$ and that the line of force is eventually recaptured by this particle after it has been once torn a way. This would account for the return of the line of force to approximately the original direction.

A further difficulty in the present theory is that when an

* The corresponding expression appropriate in Einstein's generalized theory of relativity is easily written down, but the expression for $\tau$ in terms of $x, y, z$ and. $t$ is not given by such a simple equation as (2). 
electron both rotates and revolves, different lines of force generally revolve at different rates when $a=0$ and there may be only one line of force whose angular velocity corresponds with the frequency of the light. This may, however, be the particular line of force to which we have just referred. To make all the lines of force revolve with the same angular velocity it seems necessary to make a equal to the acceleration as in Page's analysis, but then a nonradiating orbit is an impossibility.

8. According to the present theory the idea that all electromagnetic fields occurring in nature can be built up from the fields of electric poles travelling with velocities less than $c$ seems to be untenable; it seems almost necessary, in fact, to adopt a more general type of electromagnetic field as fundamental, and it may be that the simple radiant fields described by the present author will turn out to be the most suitable.

VII. The Angular Momentum and some Related Properties of the Ring Electron. By H. Stanley Allen, M.A., D.Sc., University of Edinburgh *.

\section{The Angular Momentum of the Ring Electron.}

GHORTLY before the war the late Prof. S. B. MeLaren $\checkmark$ was engaged in writing on the magneton $t$, and it is greatly to be regretted that in consequence of his death on the Western front on 14 August, 1916, the applications of his work to the theory of complete radiation, spectral series, and the asymmetrical emission of electrons in ultraviolet light were never published. Rejecting entirely the iden of magnetic or electric substance, he regarded the magneton as an inner limiting surface of the rether, formed like an anchor ring. The tubes of electric induction which terminate on its surface give it an electric charge, the magnetic tubes linked through its aperture make it a permanent magnet. $\mathrm{He}$ found that the angular momentum of any such system, whatever its shape or dimensions, about its axis of symmetry is $\left(8 \pi^{2} c\right)^{-1} \mathrm{~N}_{e} \mathrm{~N}_{m}$. Here $c$ is the velocity of light, $\mathrm{N}_{e}$ is the number of tubes of electric induction terminating on the surface, and $N_{m}$ is the number of tubes of magnetic induction passing through the aperture.

* Communicated by the Author.

† Phil. Mag. vol. xxvi. p. 800 (1913); 'Nature,' vol. xcii. p. 165 (1913), vol. xcrii. p. 547 (1916).

Phil. Mag. S. 6. Vol. 41. No. 241. Jan. 1921. 